Abhigyan Malviya, Jadran Vrabec

\title{
Henry's Law Constant of Nitrogen, Oxygen, and Argon in Ternary Aqueous Alcoholic Solvent Mixtures
}

Journal article | Accepted manuscript (Postprint)

This version is available at https://doi.org/10.14279/depositonce-9915

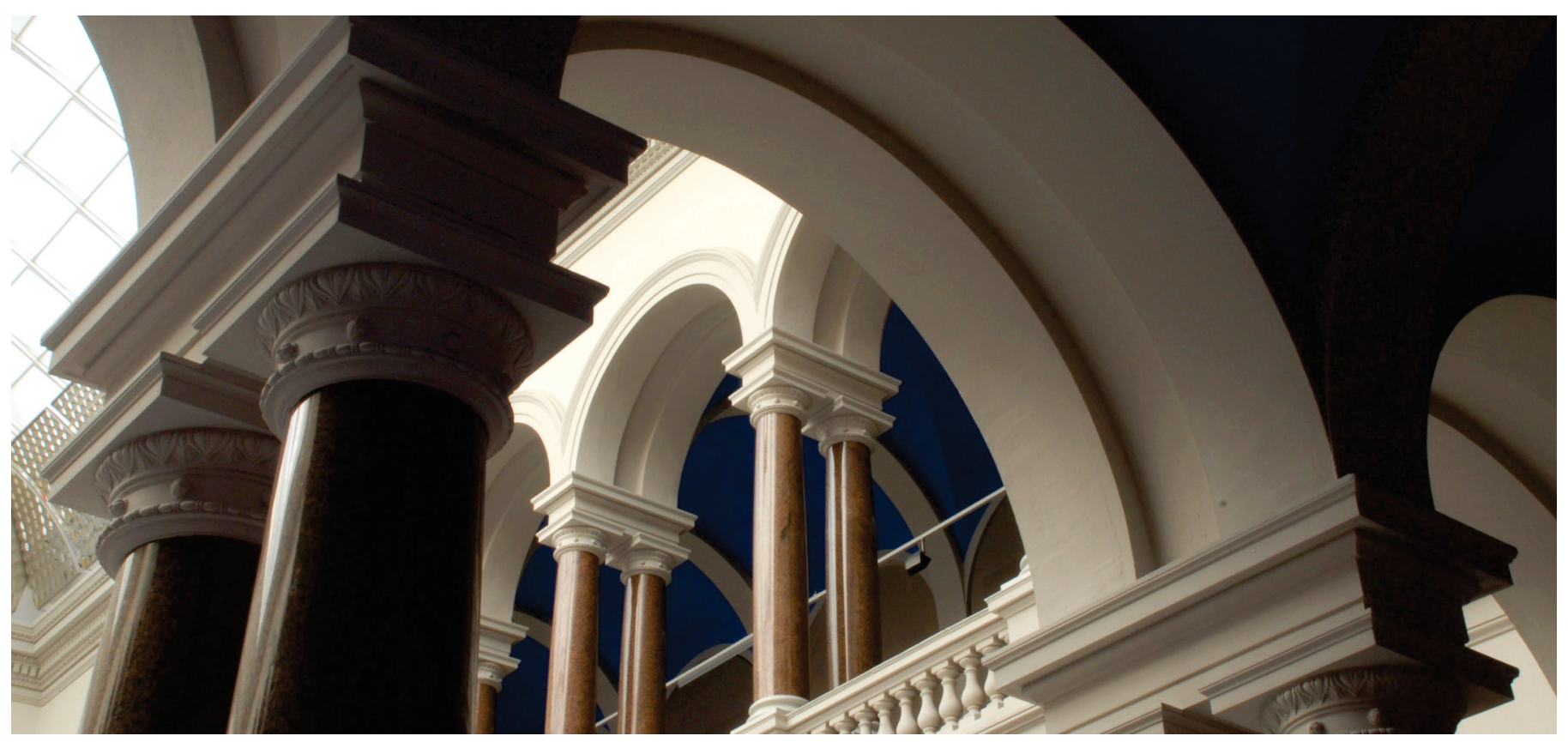

Malviya, A., \& Vrabec, J. (2019). Henry's Law Constant of Nitrogen, Oxygen, and Argon in Ternary Aqueous Alcoholic Solvent Mixtures. Journal of Chemical \& Engineering Data, 65(3), 1189-1196. https:// doi.org/10.1021/acs.jced.9b00571 


\title{
Henry's law constant of nitrogen, oxygen and argon in ternary aqueous alcoholic solvent mixtures
}

\author{
Abhigyan Malviya, Jadran Vrabec \\ Thermodynamics and Process Engineering, Technical University of Berlin, 10587 Berlin, Germany
}

\begin{abstract}
Henry's law constant data for nitrogen, oxygen and argon in the ternary liquid mixture water+methanol+ethanol as well as its pure and binary solvent subsystems are reported. The composition and temperature dependence of the Henry's law constant of the air components in these solutions is investigated by Monte Carlo simulation. The underlying molecular force field models are based on the modified Lorentz-Berthelot combining rules that contain one binary interaction parameter, which is mainly taken from preceding work. Predictions from the molecular models for the pure and binary solutions are convincingly compared to experimental literature data, where available. This finding gives confidence that the Henry's law constant for the ternary solution is predicted appropriately. Based on an extensive molecular simulation data set, correlations are established for the Henry's law constant between 274.15 and $473.15 \mathrm{~K}$.
\end{abstract}

* email: vrabec@tu-berlin.de 


\section{Introduction}

Gas solubility data are required for the understanding of natural processes as well as the design and optimization of industrial applications [1]. This ranges from gas absorption systems, over the design of reactors, to waste water treatment plants. Information on gas solubility has a wide range of uses and yet the amount of available experimental data is very limited. For the Henry's law constant of gases in water, which is typically the most important solvent, the number of systems studied experimentally is around 350 according to the Dortmund Data Bank [2]. Even after improvements of laboratory techniques over the last century, the measurement of gas solubility data is still a time consuming and laborious task. While the amount of data for pure and binary solvent systems is nonetheless significant, there are practically no experimental data for the gas solubility in ternary solvents.

Most molecular simulation studies considered gases in pure solvents, but there are few works involving binary solvents. Focus has recently been on the solubility of light gases in pure solvents. This includes solubility in eletrolytes [3-7], acids [8] and ionic liquids [9]. Some publications report computational studies to generate a better understanding about the effect of solute molecules on the solvent-solvent molecular interactions near the infinite dilution limit, but this has largely been confined to pure water as a solvent [10]. Important simulation work on ethanol as a solvent has been carried out in the context of the Industrial Fluid Properties Simulation Challenge (IFPSC) in 2004 [11], where predictions of the solubility of several gases in ethanol were made. The according contributions were published by Boutard et al. [12], Cichowski et al. [13], Wu et al. [14], Zhang and Siepmann [15] and Schnabel et al. [16]. In another work, Schnabel et al. [17], predicted the gas solubility of various solutes in methanol.

The molecular interaction between solute and solvent plays a crucial role for the Henry's law constant, while the solvent-solvent interaction indirectly influences the gas solubility. The solvent-solvent interaction is inherently defined by the solvent molecular model and was hence treated as set in this work. On the other 
hand, the solute-solvent interactions need to be specified and are often optimized to experimental data. The specification of unlike interaction parameters may add some level of difficulty into the simulation based approach

to calculate the gas solubility. Even though a lot has been invested in the past decade in the field of molecular modelling and simulation, the gas solubility in binary and ternary aqueous alcoholic mixtures has largely been left untouched. E.g., previous work of our group [18] has considered almost a hundred solute-solvent pairs with molecular simulation. More recently, Kvam and Sarkisov [19] provided a hybrid approach to obtain the gas solubility by combining pure solvent experimental data with non-ideal contributions from molecular simulation. On the other hand, we are not aware of a single publication dealing with the systematic prediction of the Henry's law constant in ternary solvents.

The motivation for the present work has been twofold. First, a detailed study on the Henry's law constant of the three air components in the aqueous alcoholic mixture water+methanol+ethanol was carried out. For that purpose, molecular force field models from preceding work, partly coauthored by Hans Hasse, were taken because they yield a very good agreement with experimental pure solvent properties. Second, correlations for the Henry's law constant of nitrogen, oxygen and argon in the pure components of that ternary solvent mixture as a function of temperature were established. Their functional form follows the work of Fernández-Prini et al. [20] that is recommended by The International Association for the Properties of Water and Steam.

\section{Methodology}

The Henry's law constant of the three solutes nitrogen, oxygen and argon in the pure solvents water, methanol and ethanol as well as their binary and ternary solvent mixtures was predicted. Binary solvents were considered with mole fractions of $0.2,0.4,0.6$ and $0.8 \mathrm{~mol} / \mathrm{mol}$. Together with the two pure solvents, this entails six compositions for one binary pair, resulting in a total of 12 binary compositions. Additional runs were performed for the aqueous binary solvents water+methanol and water+ethanol at the mole fraction $x_{\mathrm{H} 2 \mathrm{O}}=0.9 \mathrm{~mol} / \mathrm{mol}$ because the Henry's law constant varies strongly in the water-rich composition range. For ternary mixtures, the 
compositions were varied between mole fractions of $0.2,0.4$ and $0.6 \mathrm{~mol} / \mathrm{mol}$ of each component, leading to a total of six truly ternary solvent systems. Together with the three pure solvents, 23 mixtures were studied at 11 temperature levels, i.e. from $273.15 \mathrm{~K}$ to $473.15 \mathrm{~K}$ with an interval of $20 \mathrm{~K}$, resulting in a total of 253 state points.

The TIP4P/2005 [21] model was used for water, while the force field parameters for methanol were taken from Schnabel et al. [17] and for ethanol from Schnabel et al. [16]. A coarse grained approach was used for the alcohols, treating the methyl and methylene groups as a single site. As such, each solvent molecule was described by three point charges with one, two or three Lennard-Jones sites for water, methanol or ethanol, respectively. The molecular interactions of the solutes were described with one or two Lennard-Jones sites for the short-range interactions, taking parameters of Vrabec et al. [22]. Nitrogen and oxygen were modelled by two Lennard-Jones sites with a point quadrupole, while argon was treated as a single Lennard-Jones site.

For the present calculations, the molecular simulation tool $m s 2$ [23] was used. Monte Carlo sampling was carried out in the isobaric-isothermal $(N p T)$ ensemble, specifying the saturated liquid state of the solvent. A total of 864 solvent molecules was used throughout. The mole fraction of the solutes was zero to meet the infinite dilution criterion that is implied by Henry's law. Hence, solute molecules were only inserted as test particles at random coordinates and removed after their potential energy calculation. Each run consisted of $3 \cdot 10^{4}$ equilibration cycles, followed by $1.6 \cdot 10^{6}$ production cycles. The acceptance ratio was 0.5 for all Monte Carlo moves and the cutoff radius was $12.5 \AA$ throughout. The electrostatic long-range interactions were considered using the reaction field method with tin-foil boundary conditions and analytical mean field corrections employed for dispersion. Statistical uncertainties were estimated by averaging over uncorrelated blocks of 5000 cycles, employing the method of Flyvbjerg and Petersen [24].

For pure solvents, the saturation vapor pressure was specified. In case of mixtures, a linear interpolation was used to estimate the saturated vapor pressure. The methanol+ethanol mixture is largely ideal so that a linear interpolation is in order. For the aqueous alcoholic solutions, the non-ideality would mean that a larger 
pressure is observed experimentally than implied by the linear interpolation. For example, at a temperature of $323.15 \mathrm{~K}$ for the $x_{\mathrm{MeOH}}=0.1 \mathrm{~mol} / \mathrm{mol}$ aqueous methanol solution, the linearly interpolated pressure is 0.014 $\mathrm{MPa}$, while the experimental value is $0.021 \mathrm{MPa}[25]$. Although the relative difference is significant, it is hardly perceptible for liquid systems in absolute pressure terms. To further clarify this point, test simulations were performed specifying the experimental vapor pressure. The relative error in the Henry's law constant value for the two cases was around $0.3 \%$, which was typically within the statistical uncertainties of the simulation data.

\section{Henry's law constant}

The Henry's law constant was calculated from the residual chemical potential of the solute $i$ at infinite dilution $\mu_{i}^{\infty}[16,26]$

$$
H_{i}=\rho_{\mathrm{S}} k_{\mathrm{B}} T \exp \left(\mu_{i}^{\infty} /\left(k_{\mathrm{B}} T\right)\right)
$$

where $k_{B}$ is the Boltzmann constant, $T$ the temperature and $\rho_{\mathrm{S}}$ the density of the solvent in its saturated liquid state. The residual chemical potential of the solute $\mu_{i}^{\infty}$ was evaluated with Widom's test particle method [27]. The Widom method fails when the probability of successful particle insertion becomes low. In this study, 3000 test particles per cycle were inserted for the calculation of Henry's law constant, resulting in a total of $5 \cdot 10^{9}$ insertions, which gave sufficiently good statistics. The potential energy between the solute test molecule $i$ and the solvent molecules $\psi_{i}$ was sampled, leading to the residual chemical potential

$$
\mu_{i}^{\infty}=-k_{\mathrm{B}} T \ln \left\langle V \exp \left(-\psi_{i} /\left(k_{\mathrm{B}} T\right)\right)\right\rangle /\langle V\rangle
$$

where $V$ is the volume and the brackets represent the $N p T$ ensemble average.

The Henry's law constant depends on both the solvent-solvent and solute-solvent interactions. The former are fully defined by the solvent molecular model, while the latter need to be specified. The electrostatic part of the 
unlike interaction is given by laws of electrostatics for coulombic interactions, while for the unlike Lennard-Jones interaction, the modified Lorentz-Berthelot combining rules were used

$$
\sigma_{i \mathrm{~S}}=\frac{\sigma_{i}+\sigma_{\mathrm{S}}}{2}
$$

and

$$
\epsilon_{i \mathrm{~S}}=\xi \cdot \sqrt{\epsilon_{i} \epsilon_{\mathrm{S}}}
$$

where $\xi$ is a state-independent binary parameter to optimize unlike dispersion. The Henry's law constant is highly sensitive to $\xi$ and it increases with decreasing $\xi$ [16]. A larger value of $\xi$ entails a more pronounced dispersive attraction that leads to a higher solubility and hence to a lower Henry's law constant. The $\xi$ values for the solvent-solvent interactions were set to unity, assuming fully predictive liquid mixtures. For the solute-solvent interactions, the $\xi$ values were taken from preceding works or fitted to experimental data, cf. Table 1.

Table 1. Binary interaction parameter values $\xi$ were taken from preceding work or adjusted here.

\begin{tabular}{cccc}
\hline Solute & Solvent & $\xi$ & Reference \\
\hline Nitrogen & Water & 1.07 & {$[28]$} \\
Nitrogen & Methanol & 1.033 & this work \\
Nitrogen & Ethanol & 1.045 & this work \\
Oxygen & Water & 1 & {$[28]$} \\
Oxygen & Methanol & 0.988 & this work \\
Oxygen & Ethanol & 0.982 & this work \\
Argon & Water & 1.05 & {$[28]$} \\
Argon & Methanol & 1.031 & {$[17]$} \\
Argon & Ethanol & 1.044 & {$[17]$} \\
\hline
\end{tabular}




\section{Results and discussion}

\subsection{Simulation}

For 23 solvent systems constituting the aqueous alcoholic mixture water+methanol+ethanol, the Henry's law constant $H_{i}$ was calculated with molecular simulation. The full numerical data set is provided in the electronic supplementary material, together with an estimate of the statistical uncertainty.

For this discussion, the solvent mixture was grouped into three categories. The first category comprises the three pure solvents, cf. Fig. 1. Due to the fact that the binary interaction parameter was adjusted to experimental $H_{i}$ data, the data sets from simulation and experiment agree quite well. For water, the available experimental data show that the predicted Henry's law constant is mostly within range, which is particularly true for oxygen and argon. For nitrogen, the predicted values are a bit off, but this was observed only at very high temperatures and the agreement is appreciable at temperatures of up to $350 \mathrm{~K}$. For methanol, experimental values are scarce and they appear to show no clear trend so that it is hard to compare effectively with the simulation values. The binary interaction parameter $\xi$ for the pairs nitrogen-methanol and oxygen-methanol was adjusted in the present work. In both cases, it was fitted to the vapor pressure at low temperature [29-33], but at finite solute mole fractions due to the lack of reliable Henry's law constant data. For nitrogen in methanol, the Henry's law constant agrees quite well with the experimental data in Fig. 1, whereas for oxygen the agreement is not very good. However, since the variation of the experimental values from different sources at the same temperature is large, we concluded that our values are well within the uncertainty required for this work. The binary parameter $\xi$ for argon-methanol was taken from previous work [17]. For ethanol, the available experimental data base is also rather limited. Present simulation data for oxygen-ethanol agree very well with the few available experimental points, for the nitrogen-ethanol pair the agreement is well within uncertainty. The $\xi$ value was fitted to data by Friend et al. [54] in both the cases because these were assumed to be the most reliable literature data and have been used in the IFPSC as a benchmark. Similar to methanol, the binary interaction parameter for argon-ethanol was taken from Schnabel et al. [17]. Full comparison with experimental data is provided in Fig. 1. 
The individual behavior of Henry's law constant is peculiar with respect to the solvent. For example, for water, the Henry's law constant increases, reaches a distinct maximum and then decreases as the temperature rises. This behavior is associated with hydrogen bonding liquids so that methanol and ethanol also exhibit a small peak, but the variation is not that pronounced. Further, the order of magnitude and also the temperature dependence vary. $H_{i}$ ranges from around $3 \mathrm{GPa}$ for oxygen in water at $273.15 \mathrm{~K}$, increases with temperature to around $6 \mathrm{GPa}$ at $373.15 \mathrm{~K}$ and then decreases to $3 \mathrm{GPa}$ at $473.15 \mathrm{~K}$. The magnitudes of $H_{i}$ are almost double for nitrogen in water. On the other hand, for methanol and ethanol, the Henry's law constant is one order of magnitude smaller, and changes much less with temperature.

For the second category, containing the 12 binary solvent systems, some experimental data are available, but only for a few discrete temperatures. A comparison with experimental data for the water+methanol solution is shown in Fig. 2, for which Tokunaga [55] carried out experiments at temperatures of 273.15, 293.15 and 313.15 K. The experimental data of Tokunaga [55] and present simulation data agree well at the highest temperature $313.15 \mathrm{~K}$ over the entire composition range for both nitrogen and oxygen. At ambient temperature $293.15 \mathrm{~K}$, the agreement is good at higher methanol content. However, at methanol mole fractions below $0.2 \mathrm{~mol} / \mathrm{mol}$, the data provided by Tokunaga show an unexpected trend, often going through a minimum and rising again. From our point of view, such a behavior is unlikely for this aqueous alcoholic solution. The simulation data show a monotonic decrease in solubility upon addition of methanol. For oxygen, Tokunaga also measured the solubility at $273.15 \mathrm{~K}$. Again, the agreement is good at higher methanol concentrations, but similarly unexpected variations are present for $x_{\mathrm{MeOH}}<0.2 \mathrm{~mol} / \mathrm{mol}$.

In analogy to water+methanol, Tokunaga provided experimental data for the water+ethanol solution. The agreement with simulation data is again similar. For high temperatures, the agreement is good over the entire composition range, while for lower temperatures, the experimental data do not seem to be reliable, cf. Fig. 3.

While laboratory work typically leads to limited data sets, molecular simulations can be carried out in parallel, easily covering the entire range of relevant conditions. Fig. 4 shows the Henry's law constant of the three solutes 
in the binary solvent system water+methanol. An important result is the sharp increase of solubility upon addition of methanol to pure water. To further pinpoint this fact, simulations for solvent mixtures with $x_{\mathrm{MeOH}}=$ $0.1 \mathrm{~mol} / \mathrm{mol}$ were carried out. Upon addition of a slight amount of alcohol, the solubility of the gas increases by one order of magnitude. This may be attributed to the interaction between water and methanol molecules. The intermolecular hydrogen bond formation between the two solvents breaks the cage structure of hydrogen bonded water molecules. This implies more space for the solute molecules to occupy in the mixture, leading to the presence of interstitial sites and hence an increased solubility [56]. The solubility increases as more methanol is added to the system. Another important observation is the shift of the peak in the curves. For pure water, a clear maximum occurs for the Henry's law constant. However, this peak is less pronounced and shifts towards lower temperatures upon addition of methanol. These findings are qualitatively the same for all three solutes considered in this study.

The water+ethanol solution behaves similarly as the water+methanol solution, having a slighly better solubility for the present solutes, cf. Fig. 5. Again, the solubility rises sharply upon addition of a slight amount of ethanol to water. The Henry's law constant maximum shifts towards lower temperature, and the shift is more pronounced than in the water+methanol case.

As expected, the binary methanol+ethanol solvent exhibits an almost ideal mixture behavior, with the Henry's law constant value rising when methanol is added, cf. Fig. 6. This is in agreement with the pure solvent results, where methanol has a lower solubility than ethanol. Upon addition of ethanol to the solution, the solubility of the air components increases almost linearly.

For the third category, containing the ternary solvents, there are no experimental $H_{i}$ data. Because the present molecular models provide convincing results for all pure solvents as well as for four binary solvent systems that can be assessed by experimental data, it can be conjectured that the data predicted for the ternary solvent are reasonable. Ternary plots showing the solubility trends of all three air components in the water + methanol+ethanol 
solution over the entire composition range are given in Fig. 7. The ternary plots shed light on the decreased solubility of all solute molecules as the concentration of water is increased.

Another aspect that can be studied on the basis of the present simulation data is the general temperature trend of the Henry's law constant for different solutes in a given solvent. The three solutes show a remarkably similar trend in all the pure solvents and their binary and ternary solvent mixtures. Further, with increasing temperature, the data sets converge for the different solutes. Thus, it can be concluded that the Henry's law constant at high temperatures is less influenced by the solute properties through the unlike interaction, but mainly by the like solvent-solvent interaction.

\subsection{Correlation}

A correlation of the form proposed by Fernández-Prini et al. [20] was fitted to the present simulation data set for pure solvents. It was generalized for different solvents by introducing parameter $D$ because the correlation by Fernández-Prini et al. was defined for water only

$$
\ln H_{i} / p=A / T_{R}+B\left(1-T_{R}\right)^{0.355} / T_{R}+C\left(T_{R}\right)^{D} \exp \left(1-T_{R}\right) .
$$

Therein, $p$ is the vapor pressure of the solvent at the reduced temperature $T_{R}=T / T_{c}$ and $T_{c}$ is its critical temperature.

The parameters for the different binary solvent-solute pairs are provided in Table 2. Further parameters for the mole fractions considered in this work, both for binary and ternary mixtures, are made available in the supplementary material. These correlations are mostly within the small statistical uncertainty of the present simulation data. 
Table 2. Parameters $A, B, C$ and $D$ of Eq. (5) for all nine considered binary solute-solvent pairs.

\begin{tabular}{cccccc}
\hline Solute & Solvent & $A$ & $B$ & $C$ & $D$ \\
\hline Nitrogen & Water & -8.5887 & 5.1571 & 10.3910 & -0.42 \\
Nitrogen & Methanol & 3.4197 & 5.0269 & -2.2366 & 0.77 \\
Nitrogen & Ethanol & 5.0932 & 4.2677 & -3.8154 & 0.79 \\
Oxygen & Water & -7.8612 & 4.8515 & 9.6698 & -0.42 \\
Oxygen & Methanol & 3.3816 & 4.6089 & -2.1826 & 0.77 \\
Oxygen & Ethanol & 5.0873 & 3.9010 & -3.7743 & 0.79 \\
Argon & Water & -7.9964 & 4.8367 & 9.7484 & -0.42 \\
Argon & Methanol & 3.4892 & 4.4650 & -2.2961 & 0.77 \\
Argon & Ethanol & 5.1636 & 3.6970 & -3.8754 & 0.79 \\
\hline
\end{tabular}

\section{Conclusion}

It was shown that molecular simulation is a reliable method for investigating the Henry's law constant of gases dissolved in liquid solvents. To verify this issue, an extensive simulation effort was made to cover 23 mixtures in a combinatorial way, ranging from pure, over binary to ternary solvent mixtures. The employed molecular models in many cases oversimplify the molecular features of the substance that they represent. However, it was found that they are usually able to compensate such simplifications and adequately cover the gas solubility effects.

To optimally represent the phase behavior of all studied solute-solvent pairs, the unlike dispersive energy was adjusted to a single experimental Henry's law constant or the binary vapor pressure of each mixture. Based on these mixture models, the temperature dependence of the Henry's law constant was predicted and compared to the available experimental data. For the large majority of systems that can be assessed in this sense, a good agreement was found.

Furthermore, it was shown that the models are generally capable of yielding consistent Henry's law constant data for the ternary water+methanol+ethanol solvent mixture. For high temperatures, it was found for a given solvent that the Henry's law constant of different solutes converges to a narrow band. This indicates that this thermophysical property is then mainly determined by the solvent-solvent interaction. It is also clear that more experimental work is required in ternary mixtures to validate our findings. 


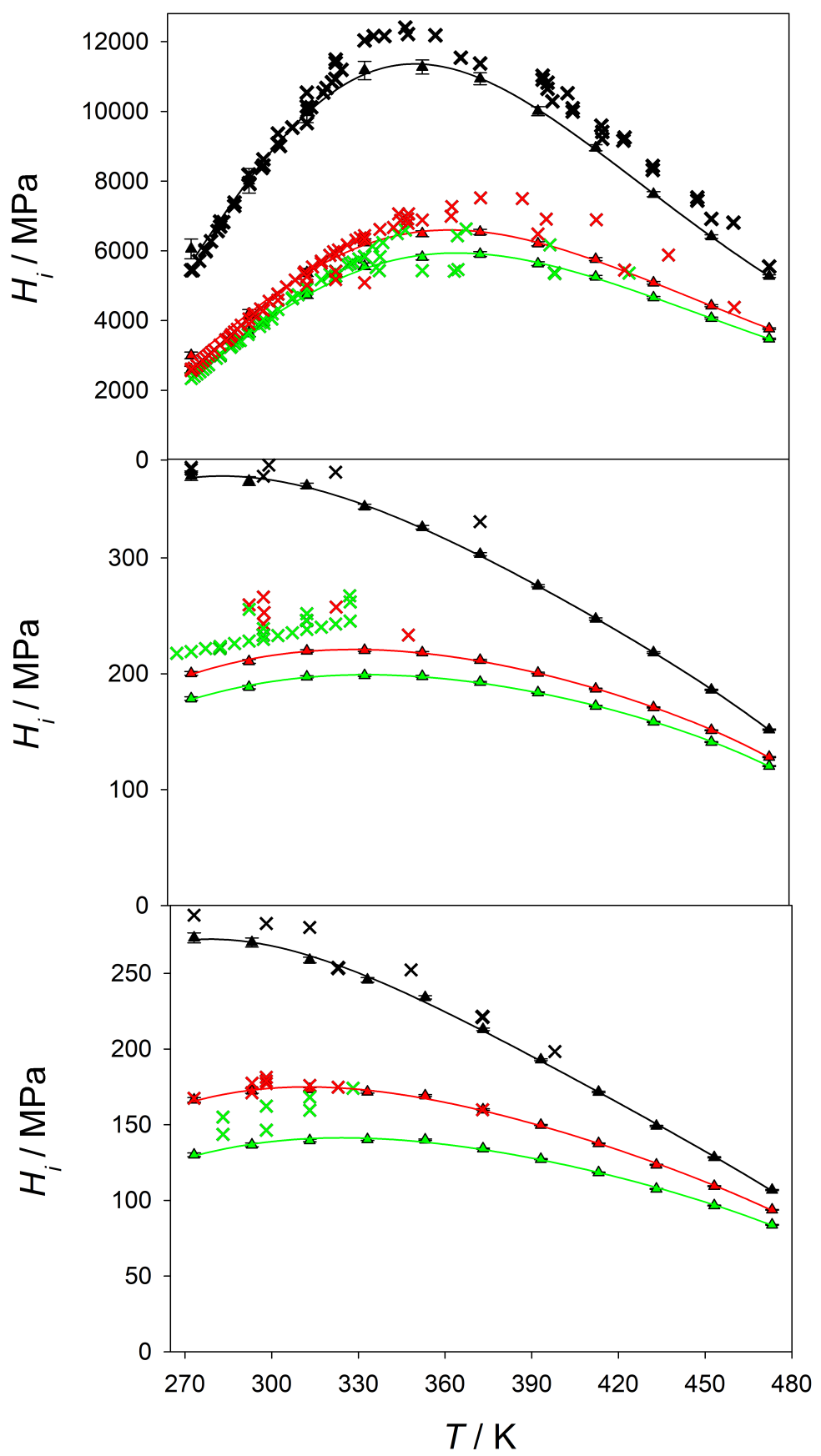

Fig. 1. Henry's law constant of $\mathrm{N}_{2}(\boldsymbol{\Delta}), \mathrm{O}_{2}(\boldsymbol{\Delta})$ and $\mathrm{Ar}(\boldsymbol{\Delta})$ in water (top), methanol (center) and ethanol (bottom). Solid lines represent correlation (5) and crosses are experimental literature data [34-53]. 


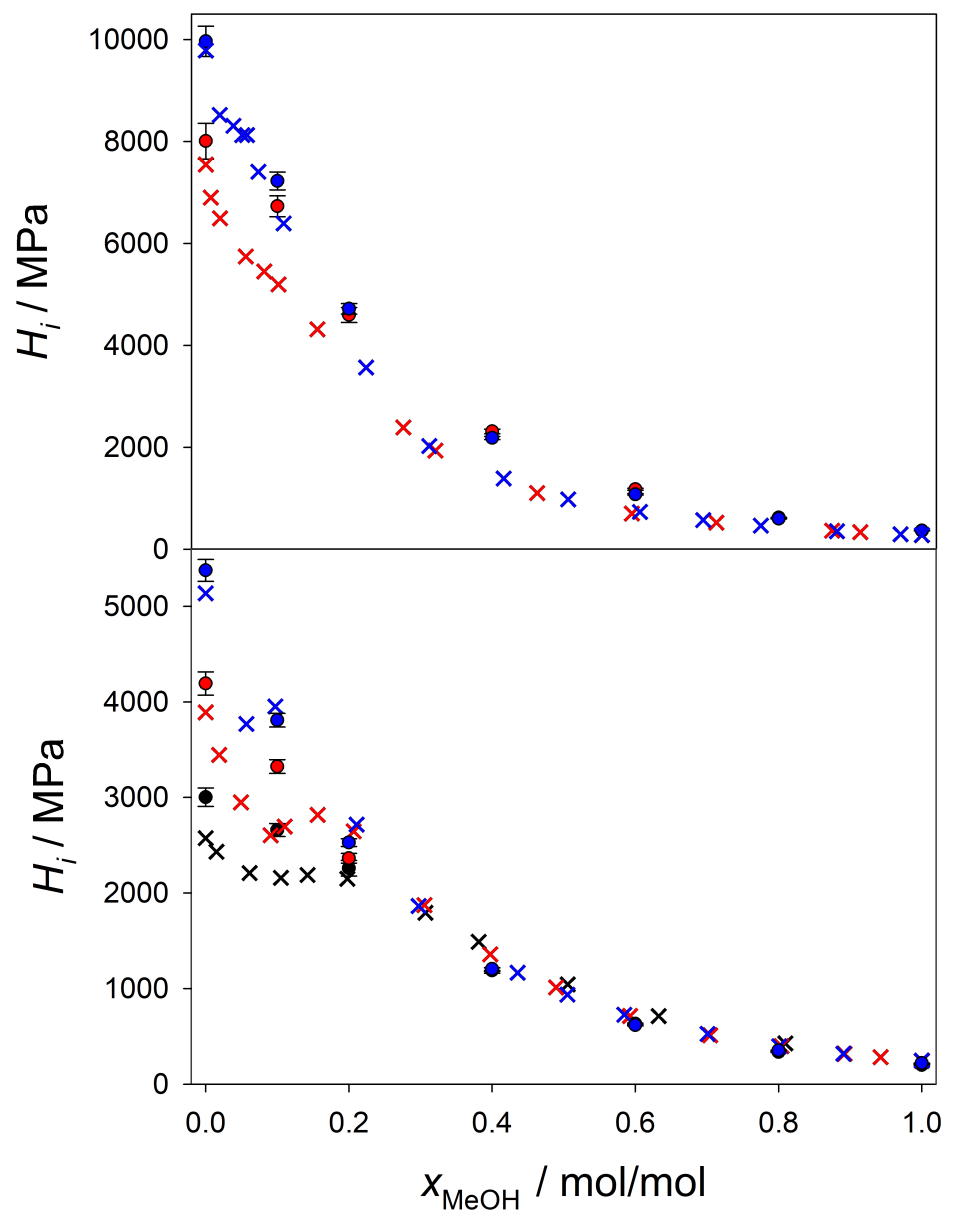

Fig. 2. Henry's law constant of nitrogen (top) and oxygen (bottom) in water+methanol at $273.15 \mathrm{~K}(\bullet), 293.15 \mathrm{~K}(\bullet)$ and $313.15 \mathrm{~K}(\bullet)$. Crosses indicate experimental data by Tokunaga [55]. 


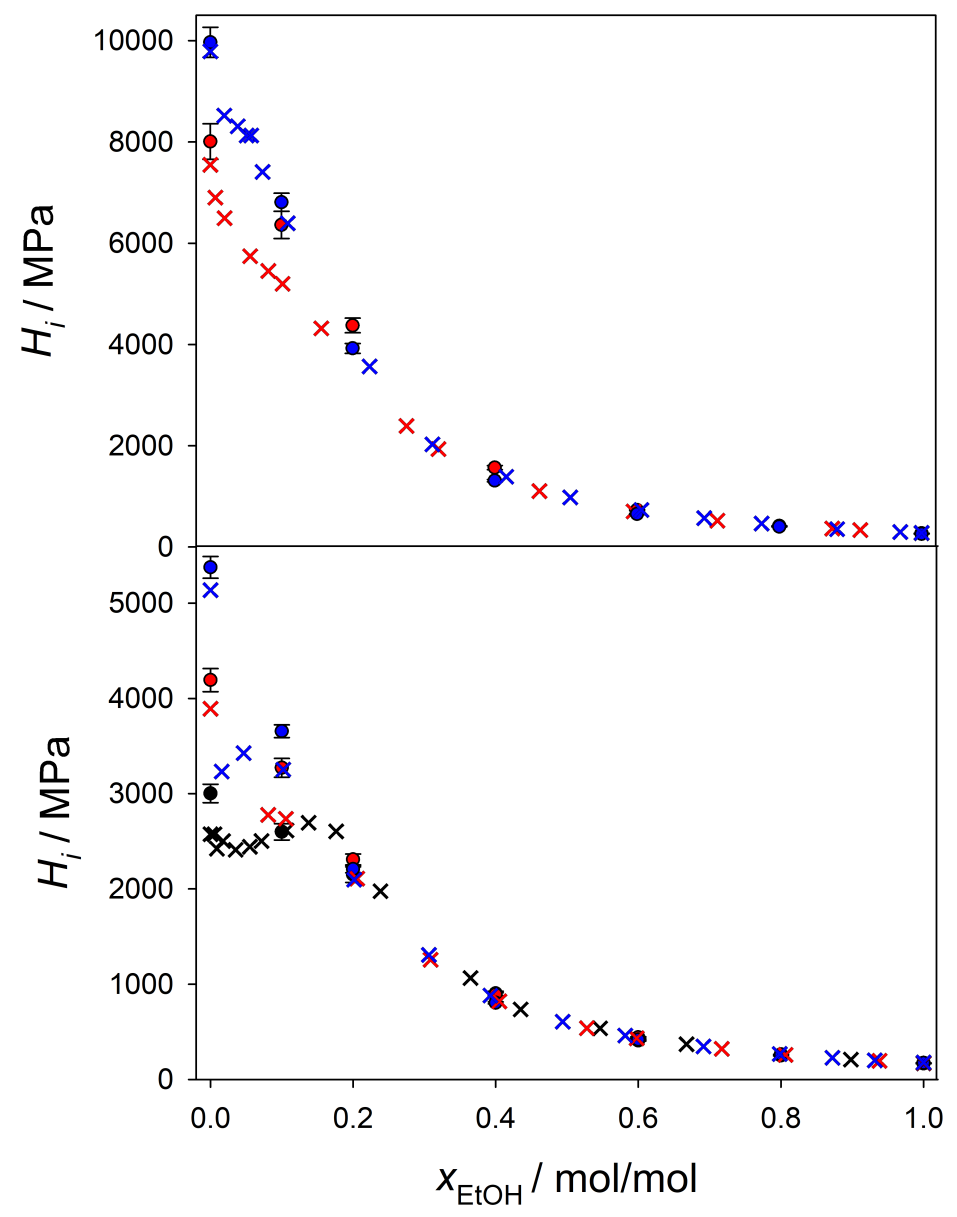

Fig. 3. Henry's law constant of nitrogen (top) and oxygen (bottom) in water+ethanol at $273.15 \mathrm{~K}(\bullet), 293.15 \mathrm{~K}(\bullet)$ and $313.15 \mathrm{~K}(\bullet)$. Crosses indicate experimental data by Tokunaga [55]. 


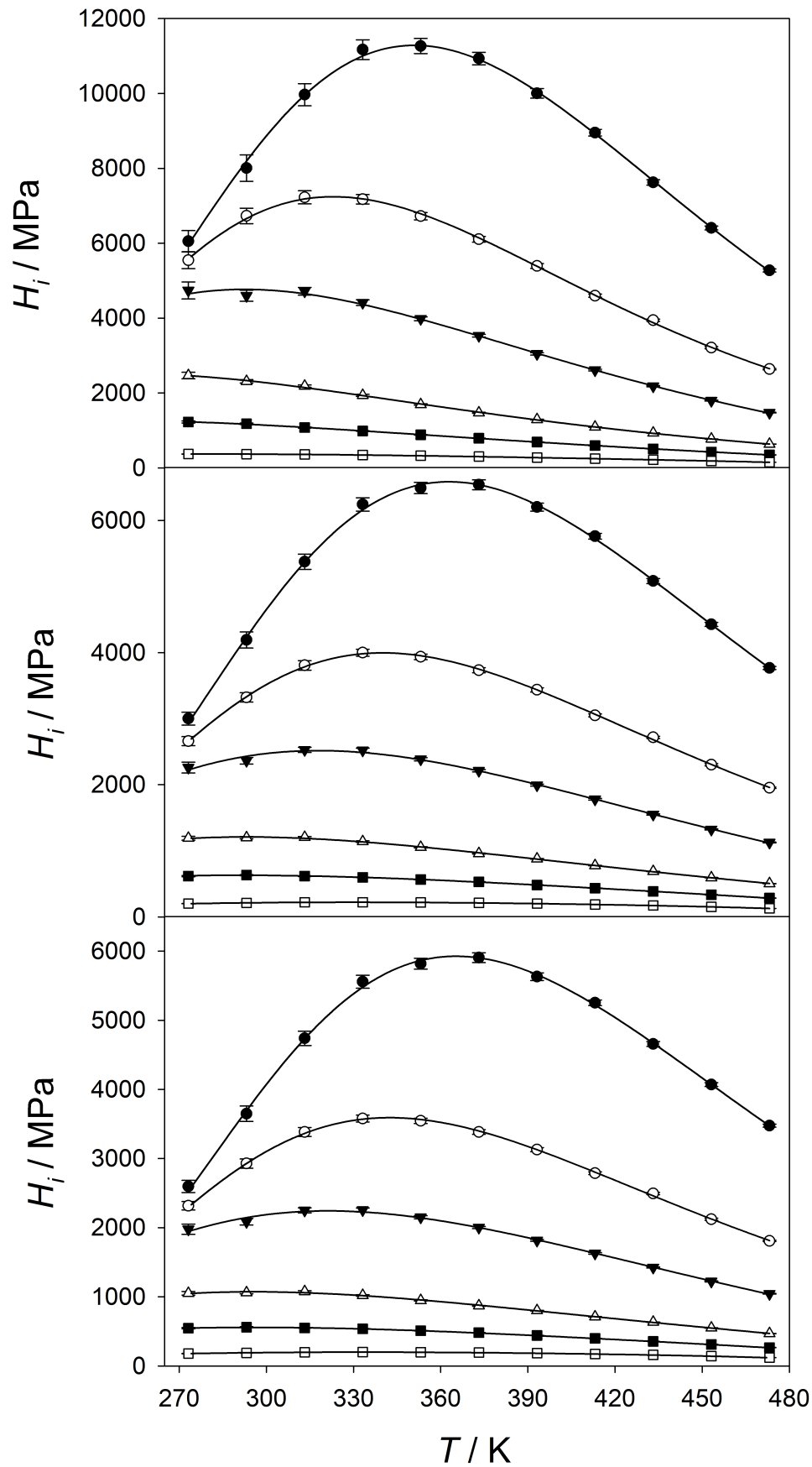

Fig. 4. Henry's law constant of nitrogen (top), oxygen (center) and argon (bottom) in water+methanol with varying mole fraction $x_{\mathrm{MeOH}}=0(\bullet), 0.1(\circ), 0.2(\mathbf{v}), 0.4(\triangle), 0.6(\mathbf{\square})$ and $1(\square) \mathrm{mol} / \mathrm{mol}$. Solid lines represent correlation (5). 


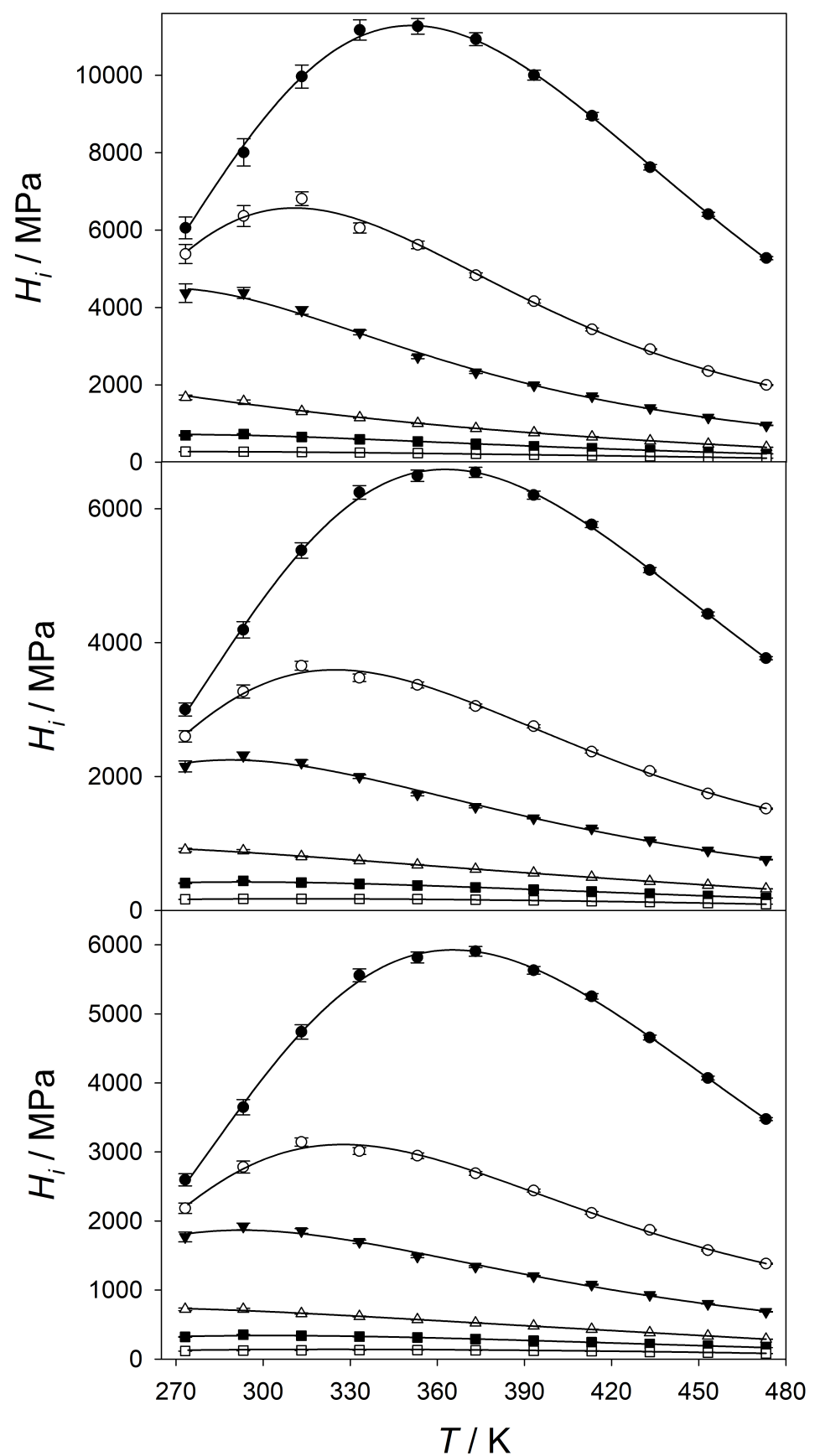

Fig. 5. Henry's law constant of nitrogen (top), oxygen (center) and argon (bottom) in water+ethanol with varying mole fraction $x_{\mathrm{EtOH}}=0(\bullet), 0.1(\circ), 0.2(\boldsymbol{\nabla}), 0.4(\triangle), 0.6(\boldsymbol{\square})$ and $1(\square) \mathrm{mol} / \mathrm{mol}$. Solid lines represent correlation $(5)$. 


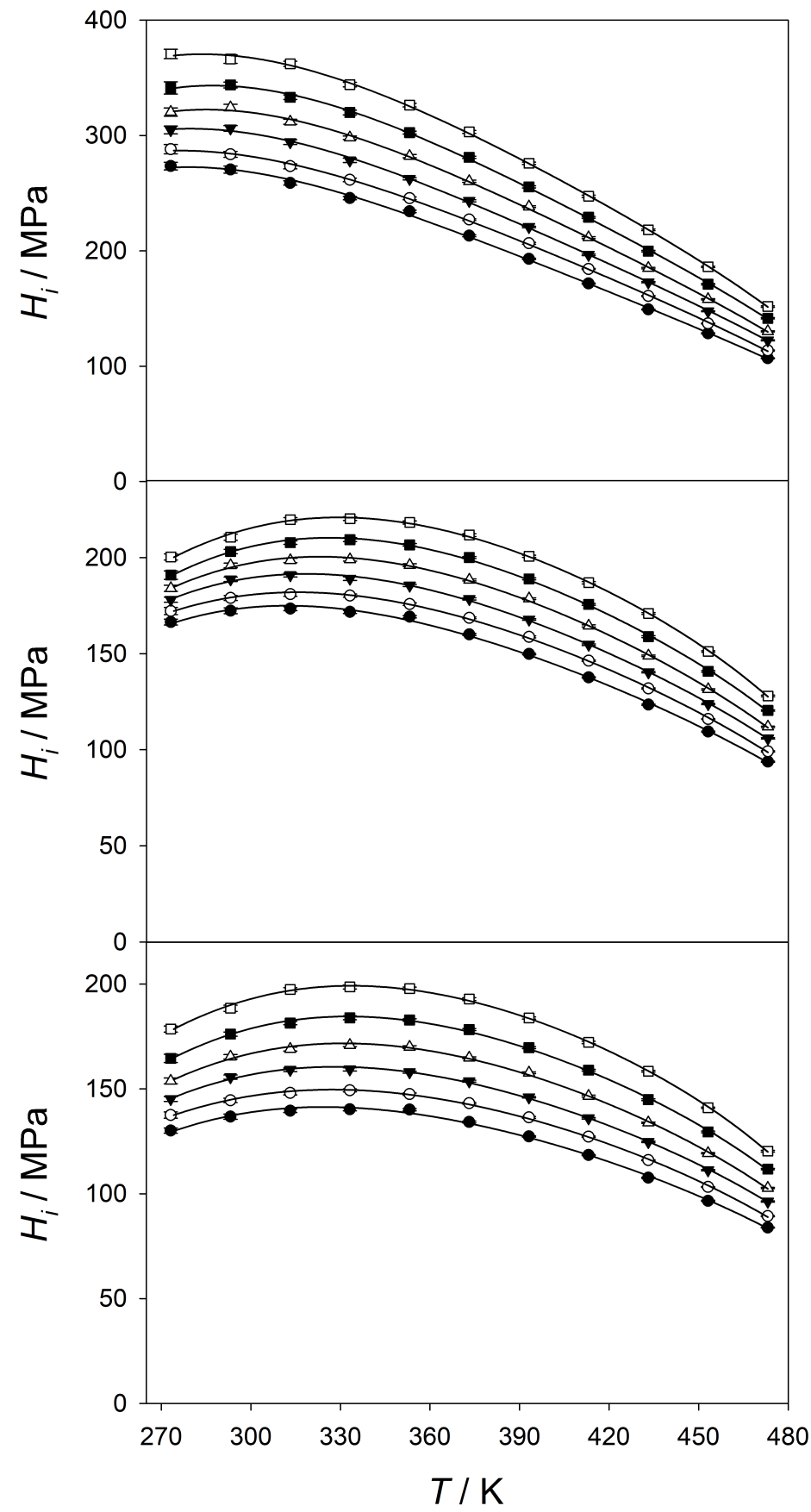

Fig. 6. Henry's law constant of nitrogen (top), oxygen (center) and argon (bottom) in methanol+ethanol with varying mole fraction $x_{\mathrm{MeOH}}=0(\bullet), 0.2(\circ), 0.4(\boldsymbol{\nabla}), 0.6(\triangle), 0.8(\boldsymbol{\square})$ and $1(\square) \mathrm{mol} / \mathrm{mol}$. Solid lines represent correlation $(5)$. 

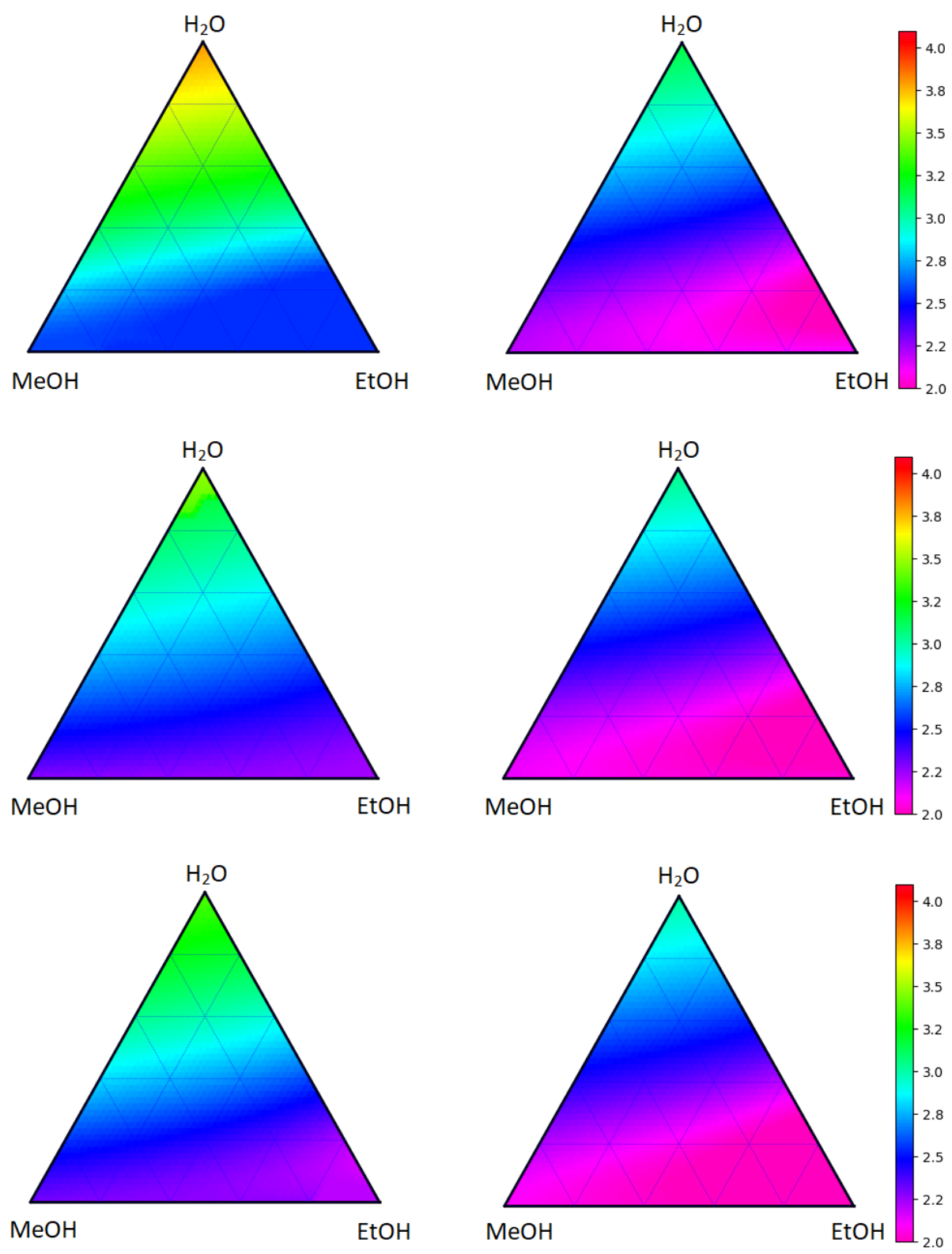

Fig. 7. Gibbs triangle plot showing the Henry's law constant (at log scale) of nitrogen (top), oxygen (center) and argon (bottom) in water+methanol+ethanol over mole fractions at temperatures of $274.15 \mathrm{~K}$ (left) and $473.15 \mathrm{~K}$ (right). 


\section{Acknowledgements}

This research was funded by BMBF under the grant 01IH16008 "TaLPas: Task-basierte Lastverteilung und Auto-Tuning in der Partikelsimulation" and computational support was given by the High Performance Computing Center Stuttgart (HLRS) under the grant MMHBF2. Furthermore, we gratefully acknowledge the Paderborn Center for Parallel Computing $\left(\mathrm{PC}^{2}\right)$ for the generous allocation of computer time on the OCuLUS and Noctua clusters. 


\title{
Supporting Information
}

\section{Henry's law constant of nitrogen, oxygen and argon in ternary aqueous alcoholic solvent mixtures}

\author{
Abhigyan Malviya, Jadran Vrabec* \\ Thermodynamics and Process Engineering, Technical University of Berlin, 10587 Berlin, Germany \\ *Corresponding author. E-mail address: vrabec@tu-berlin.de;
}

\section{Supporting Material}

\section{List of content}

Henry's law constant for pure solvents

Henry's law constant for the binary solvent water+methanol

Henry's law constant for the binary solvent water+ethanol

Henry's law constant for the binary solvent methanol+ethanol

Henry's law constant for the ternary solvent water+methanol+ethanol 


\section{References}

1. Letcher, T. M. (Ed.), Developments and Applications in Solubility; The Royal Society of Chemistry: Cambridge, 2007.

2. Dotmunder Datenbank, Mixture Properties, Version 2017.

3. Jiang, H.; Economou, I. G.; Panagiotopoulos, A. Z. Molecular Modeling of Thermodynamic and Transport Properties for CO2 and Aqueous Brines. Acc. Chem. Res. 2017, 50, 751-758.

4. Fauve, R.; Guichet, X.; Lachet, V.; Ferrando, N. Prediction of H2S solubility in aqueous NaCl solutions by molecular simulation. J. Pet. Sci. Eng. 2017, 157, 94-106.

5. Tsai, E. S.; Jiang, H.; Panagiotopoulos, A. Z. Monte Carlo simulations of H2O-CaCl2 and H2O-CaCl2-CO2 mixtures. Fluid Phase Equilib. 2016, 407, 262-268.

6. Lopez-Lazaro, C.; Bachaud, P.; Moretti, I.; Ferrando, N. Predicting the phase behavior of hydrogen in NaCl brines by molecular simulation for geological applications. BSGF - Earth Sci. Bull. 2019, 190, 7-21.

7. Creton, B.; Nieto-Draghi, C.; De Bruin, T.; Lachet, V.; El Ahmar, E.; Valtz, A.; Coquelet, C.; Lasala, S.; Privat, R.; Jaubert, J. Thermodynamic study of binary systems containing sulphur dioxide and nitric oxide: Measurements and modelling. Fluid Phase Equilib. 2018, 461, 84-100.

8. Ramdin, M.; Jamali, S. H.; van den Broeke, L. J. P.; Buijs, W.; Vlugt, T. J. H. CO2 solubility in small carboxylic acids: Monte Carlo simulations and PC-SAFT modeling. Fluid Phase Equilib. 2018, 458, 1-8.

9. Jamali, S. H.; Ramdin, M.; Becker, T. M.; Torres-Knoop, A.; Dubbeldam, D.; Buijs, W.; Vlugt, T. J. H. Solubility of sulfur compounds in commercial physical solvents and an ionic liquid from Monte Carlo simulations. Fluid Phase Equilib. 2017, 433, 50-55.

10. Boulougouris, G. C.; Voutsas, E. C.; Economou, I. G.; Theodorou, D. N.; Tassios, D. P. Henry's Constant Analysis for Water and Nonpolar Solvents from Experimental Data, Macroscopic Models, and Molecular Simulation. J. Phys. Chem. B 2001, 105, 7792-7798.

11. Case, F.; Chaka, A.; Friend, D. G.; Frurip, D.; Golab, J.; Gordon, P.; Johnson, R.; Kolar, P.; Moore, J.; Mountain, R. D.; Olson, J.; Ross, R.; Schiller, M. The second industrial fluid properties simulation challenge. Fluid Phase Equilib. 2005, 236, 1-14.

12. Boutard, Y.; Ungerer, Ph.; Teuler, J. M.; Ahunbay, M. G.; Sabater, S. F.; Pérez-Pellitero, J.; Mackie, A. D.; Bourasseau, E. Extension of the anisotropic united atoms intermolecular potential to amines, amides and alkanols. Application to the problems of the 2004 Fluid Simulation Challenge. Fluid Phase Equilib. 2005, 236, 25-41.

13. Cichowski, E. C.; Schmidt, T. R.; Errington, J. R. Determination of Henry's law constants through transition matrix Monte Carlo simulation. Fluid Phase Equilib. 2005, 236, 58-65.

14. Wu, C.; Li, X.; Dai, J.; Sun, H. Prediction of Henry's law constants of small gas molecules in liquid ethylene oxide and ethanol using force field methods. Fluid Phase Equilib. 2005, 236, 66-77.

15. Zhang, L.; Siepmann, I. Direct calculation of Henry's law constants from Gibbs ensemble Monte Carlo simulations: nitrogen, oxygen, carbon dioxide and methane in ethanol. J. Theor. Chem. Acc. 2006, 115, 391-397.

16. Schnabel, T.; Vrabec, J.; Hasse, H. Henry's law constants of methane, nitrogen, oxygen and carbon dioxide in ethanol from 273 to 498 K: Prediction from molecular simulation. Fluid Phase Equilib. 2005, 233, 134-143.

17. Schnabel, T.; Vrabec, J.; Hasse, H. Molecular simulation study of hydrogen bonding mixtures and new molecular models for mono- and dimethylamine. Fluid Phase Equilib. 2008, 263, 144-159.

18. Huang, Y.; Miroshnichenko, S.; Hasse, H.; Vrabec, J. Henry's Law Constant from Molecular Simulation: A Systematic Study of 95 Systems. Int J Thermophys. 2009, 30, 1791-1810.

19. Kvam, O.; Sarkisov, L. Solubility prediction in mixed solvents: A combined molecular simulation and experimental approach. J. Phys. Chem. B 2019, 484, 26-37.

20. Fernández-Prini, R.; Alvarez, J. L.; Harvey, A. H. Henry's Constants and Vapor-Liquid Distribution Constants for Gaseous Solutes in H2O and D2O at High Temperatures. J. Phys. Chem. Ref. Data 2003, 32, 903-916.

21. Abascal, J. L. F.; Vega, C. A general purpose model for the condensed phases of water: TIP4P/2005. J. Chem. Phys. 2005, 123, 234505.

22. Vrabec, J.; Stoll, J.; Hasse, H. A Set of Molecular Models for Symmetric Quadrupolar Fluids. Fluid Phase Equilib. 2001, 105, 12126-12133.

23. Rutkai, G.; Köster, A.; Guevara-Carrion, G.; Janzen, T.; Schappals, M.; Glass, C. W.; Bernreuther, M.; Wafai, A.; Stephan, S.; Kohns, M.; Reiser, S.; Deublein, S.; Horsch, M.; Hasse, H.; Vrabec, J. ms2: A molecular simulation tool for thermodynamic properties, release 3.0. Comput. Phys. Commun. 2017, 221, 343-351.

24. Flyvbjerg, H.; Petersen, H. G. Error estimates on averages of correlated data. J. Chem. Phys. 1989, 91, $461-466$. 
25. Kurihara, K.; Minoura, T.; Takeda, K.; Kojima, K. Isothermal vapor-liquid equilibria for methanol+ ethanol+ water, methanol+ water, and ethanol+ water. J. Chem. Eng. Data 1995, 40, 679-684.

26. Shing, K. S.; Gubbins, K. E.; Lucas, K. Henry constants in non-ideal fluid mixtures: computer simulation and theory. Mol. Phys. 1988, 65, 1235-1252.

27. Widom, B. Some topics in the theory of fluids. J. Chem. Phys. 1963, 39, 2808-2812.

28. Köster, A.; Thol, M.; Vrabec, J. Molecular models for the hydrogen age: hydrogen, nitrogen, oxygen, argon, and water. J. Chem. Eng. Data 2018, 63, 305-320.

29. Brunner, E.; Hültenschmidt, W.; Schlichthärle, G. Fluid mixtures at high pressures IV. Isothermal phase equilibria in binary mixtures consisting of (methanol + hydrogen or nitrogen or methane or carbon monoxide or carbon dioxide). J. Chem. Thermodyn. 1987, 19, 273-291.

30. Laursen, T.; Andersen, S. I. High-Pressure Vapor-Liquid Equilibrium for Nitrogen + Methanol. J. Chem. Eng. Data 2002, 47, 1173-1174.

31. Krichevskii, I. R.; Efremova, G. D. Fazovye I obemnye sootnosheniya v sistemakh zhidkost gaz pri vysoskikh davleniyakh. 3. Zh. Fiz. Khim. 1951, 25, 577-583.

32. Hemmaplardh, B. ; King Jr, A. D. Solubility of Methanol in Compressed Nitrogen, Argon, Methane, Ethylene, Ethane, Carbon Dioxide, and Nitrous Oxide. Evidence for Association of Carbon Dioxide with Methanol in the Gas Phase. J. Phys. Chem. 1972, 76, 2170-2175.

33. Fischer, K.; Wilken, M. Experimental determination of oxygen and nitrogen solubility in organic solvents up to 10 MPa at temperatures between $298 \mathrm{~K}$ and $398 \mathrm{~K}$. J. Chem. Thermodyn. 2001, 33, 1285-1308.

34. Orcutt, F. S. ; Seevers, M. H. A method for determining the solubility of gases in pure liquids or solutions by the Van Slyke-Neill manometric apparatus. J. Biol. Chem. 1937, 117, 501-508.

35. Winkler, L. W. Gesetzmässigkeit bei der Absorption der Gase in Flüssigkeiten. Z. Phys. Chem. (Leipzig) 1906, 55, 344-354.

36. Murray, C. N.; Riley, J. P.; Wilson T. R. S. (Ed.). Deep Sea Research and Oceanographic Abstracts; Elsevier: Amsterdam, 1969.

37. Wiebe, R.; Gaddy, V. L.; Heins, C. The solubility of nitrogen in water at 50, 75 and 100 from 25 to 1000 atmospheres. J. Am. Chem. Soc. 1933, 55, 947-953.

38. Cosgrove, B. A.; Walkley, J. Solubilities of gases in H2O and 2H2O. J. Chromatogr A. 1981, 216, $161-167$.

39. Saddington, A. W.; Krase, N. W. Vapor-liquid equilibria in the system nitrogen-water. J. Am. Chem. Soc. 1934, 56, 353-361.

40. Alvarez, J.; Crovetto, R.; Fernández-Prini, R. The Dissolution of N2 and of H2 in Water from Room Temperature to 640 K. Ber. Bunenges. Phys. Chem. 1988, 92, 935-940.

41. Fox, C. J. J. On the coefficients of absorption of nitrogen and oxygen in distilled water and sea-water, and of atmospheric carbonic acid in sea-water. Trans. Faraday Soc. 1909, 5, 68-86.

42. Tsuji, K.; Ichikawa, K.; Yamamoto, H.; Tokunaga, J. Solubilities of Oxygen and Nitrogen in Acetone-Water Mixed Solvent. Kagaku Kogaku Ronbun. 1987, 13, 825-830.

43. Morrison, T. J.; Billett, F. The salting-out of non-electrolytes. Part II. The effect of variation in non-electrolyte. J. Chem. Soc. 1952, 51, 3819-3822.

44. Benson, B. B.; Krause, D.; Peterson, M. A. The solubility and isotopic fractionation of gases in dilute aqueous solution. I. Oxygen. J. Solution Chem. 1979, 8, 655-690.

45. Naim, A. B.; Baer, S. Method for measuring solubilities of slightly soluble gases in liquids. Trans. Faraday Soc. 1963, 59, 2735-2738.

46. R. Crovetto, R. Fernández-Prini, M. L. Japas, Solubilities of inert gases and methane in $\mathrm{H} 2 \mathrm{O}$ and in D2O in the temperature range of 300 to $600 \mathrm{~K}$. J. Chem. Phys. 1982, 76, 1077-1086.

47. Smith, S. P.; Kennedy, B. M. The solubility of noble gases in water and in NaCl brine. Geochim. Cosmochim. Acta 1983, 47, 503-515.

48. Krause, D.; Benson, B. B. The solubility and isotopic fractionation of gases in dilute aqueous solution. IIa. Solubilities of the noble gases. J. Solution Chem. 1989, 18, 823-873.

49. Weber, W.; Zeck, S.; Knapp, H. Gas solubilities in liquid solvents at high pressures: apparatus and results for binary and ternary systems of N2, CO2 and CH3OH. Fluid Phase Equilib. 1984, 18, 253-278.

50. Luehring, P.; Schumpe, A. Gas solubilities (hydrogen, helium, nitrogen, carbon monoxide, oxygen, argon, carbon dioxide) in organic liquids at 293.2 K. J. Chem. Eng. Data 1989, 34, 250-252.

51. Fischer, K.; Wilken, M. Experimental determination of oxygen and nitrogen solubility in organic solvents up to 10 MPa at temperatures between $298 \mathrm{~K}$ and $398 \mathrm{~K}$. J. Chem. Thermodyn. 2001, 33, 1285-1308. 
52. Prorokov, V. N.; Dolotov, V. V.; Krestov, G. A. Solubility and thermodynamic characteristics for the dissolution of argon, krypton, and xenon in water and in monohydric alcohols. Russ. J. Phys. Chem. 1984, 58, $1153-1154$.

53. Nitta, T.; Fujio, J.; Katayama, T. Solubilities of nitrogen in binary solutions. Mixtures of ethanol with benzene, ethyl acetate, and diethyl ether. J. Chem. Eng. Data 1978, 23, 157-159.

54. Friend, D. G.; Frurip, D. J.; Lemmon, E. W.; Morrison, R. E.; Olson, J. D.; Wilson, L. C. Establishing benchmarks for the second industrial fluids simulation challenge. Fluid Phase Equilib. 2005, 236, 15-24.

55. Tokunaga, J. Solubilities of oxygen, nitrogen, and carbon dioxide in aqueous alcohol solutions. J. Chem. Eng. Data 1975, 20, 41-46.

56. Dalmolin, I.; Skovroinski, E.; Biasi, A.; Corazza, M. L.; Dariva, C.; Oliveira, J. V. Solubility of carbon dioxide in binary and ternary mixtures with ethanol and water. Fluid Phase Equilib. 2006, 245, 193-200. 
Table of Content

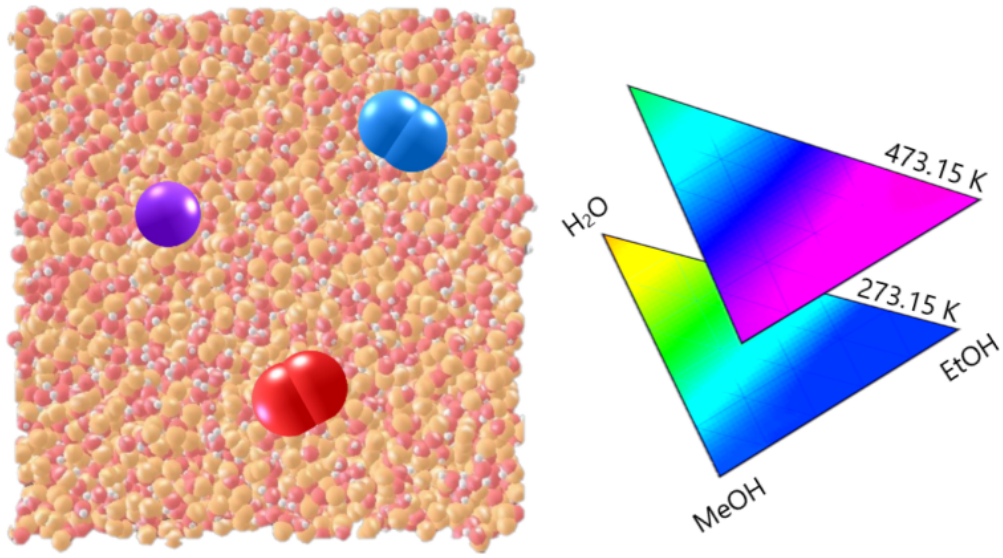

Fig. 8. Table of content 